Document downloaded from:

http://hdl.handle.net/10251/150054

This paper must be cited as:

Calvo Galve, N.; Giménez-Marqués, M.; Palomino Roca, M.; Valencia Valencia, S.; Rey Garcia, F.; Mínguez Espallargas, G.; Coronado, E. (2016). Isostructural compartmentalized spin-crossover coordination polymers for gas confinement. Inorganic Chemistry Frontiers. 3(6):808-813. https://doi.org/10.1039/C5QI00277J

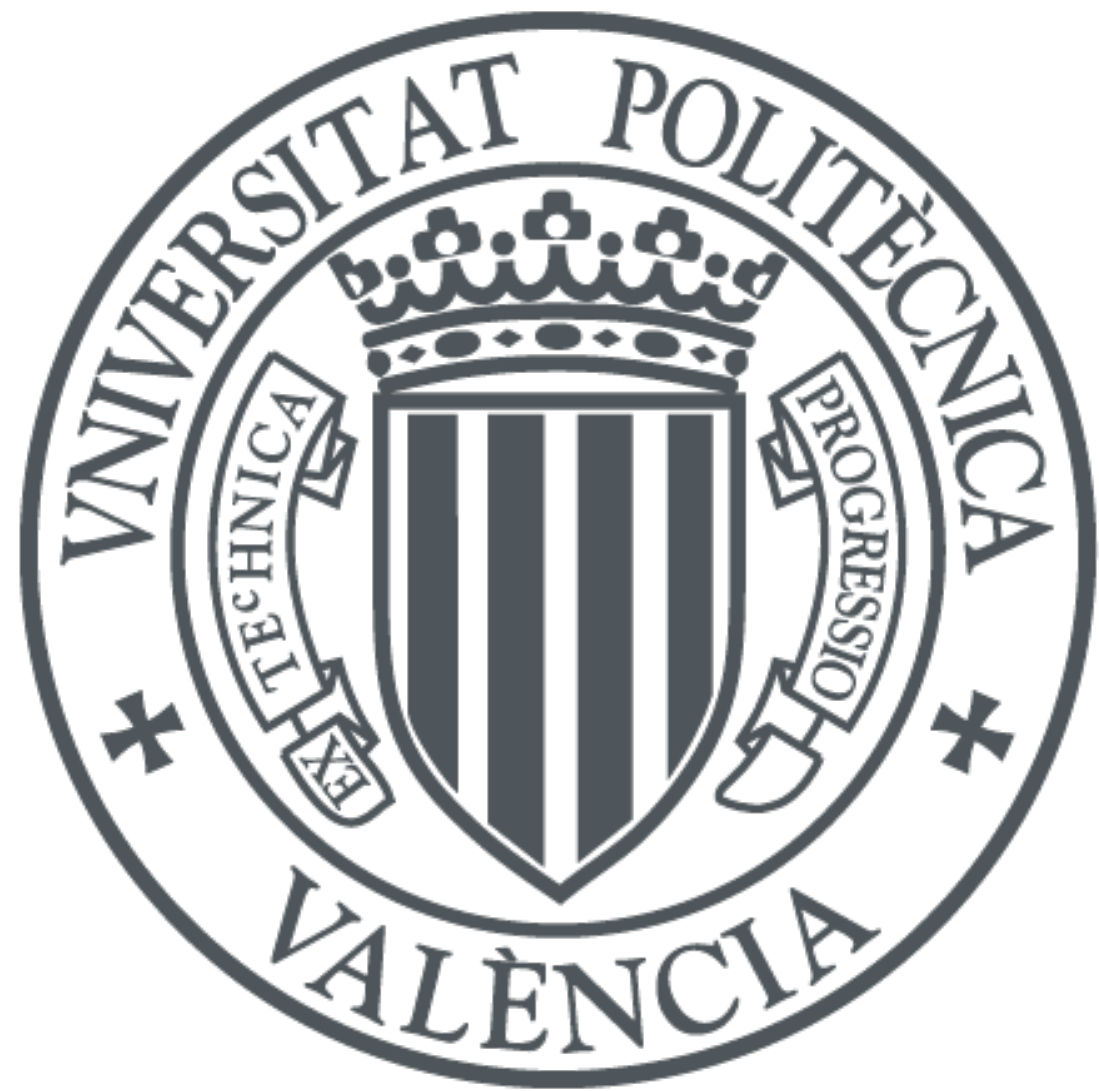

The final publication is available at

https://doi.org/10.1039/C5QI00277J

Copyright The Royal Society of Chemistry

Additional Information 


\section{Isostructural compartmentalized spin-crossover coordination polymers for gas confinement}

Received 00th January 20xx Accepted 00th January 20xx

DOI: $10.1039 / x 0 x \times 00000 x$

www.rsc.org/

\author{
Néstor Calvo Galve, ${ }^{a}$ Mónica Giménez-Marqués, ${ }^{\mathrm{a}, \mathrm{b}}$ Miguel Palomino, ${ }^{\mathrm{c}}$ Susana Valencia, ${ }^{\mathrm{c}}$ Fernando \\ Rey, ${ }^{c}$ Guillermo Mínguez Espallargas*a and Eugenio Coronado ${ }^{a}$
}

Here we present two $\mathrm{Fe}^{\prime \prime}$ coordination polymers that possess discrete compartments suitable for $\mathrm{CO}_{2}$ physisorption despite the lack of permanent channels. The two crystalline materials, of general formula $\left[\mathrm{Fe}(\mathrm{btzbp})_{3}\right](\mathrm{X})_{2}\left(\mathrm{X}=\mathrm{ClO}_{4}\right.$ or $\left.\mathrm{BF}_{4}\right)$, present voids of $c a$. $250 \AA^{3}$, which each can accommodate up to two $\mathrm{CO}_{2}$ molecules. The abrupt spin transition can be modified upon $\mathrm{CO}_{2}$ sorption, and different magnetic behaviour is observed depending on the number of molecules sorbed.

\section{Introduction}

Coordination polymers represent an important area of coordination chemistry with foremost applications in catalysis, magnetism, electronics and optics. In the last two decades the study of coordination polymers, ${ }^{1}$ which are formed by metal centres linked by organic ligands, has experienced a substantial progress and enormous research effort has focused on the synthesis and study of novel structures with singular chemical and physical properties. ${ }^{2}$ Among these, particular interest has been attracted to those that present permanent channels and large voids, thus allowing the accommodation of guest molecules such as solvents or gases. These porous coordination polymers, also known as metal-organic frameworks (MOFs), ${ }^{3,4}$ are crystalline solids that allow the combination of porosity and other physical properties typical of dense coordination polymers, with applications as sensors, ${ }^{5}$ magnetism, ${ }^{6}$ separation $^{7}$ and storage ${ }^{8}$ of gases, or drug delivery, ${ }^{9}$ among others.

Another area of much interest within the field of coordination chemistry is the study of coordination cages. ${ }^{10}$ These discrete charged units, of many varied stoichiometry, present the same structural basis than coordination polymers, that is, the coordination of organic linkers and metal centers albeit with the formation of soluble materials with an ample range of host-guest chemistry.

These two flourish areas of coordination chemistry have been combined for the first time by Fujita and co-workers, ${ }^{11}$ who have transferred the host-guest chemistry of coordination cages into the crystalline state by synthesising a porous MOF composed of $\mathrm{M}_{6} \mathrm{~L}_{4}$ octahedral molecular cages which has allowed the determination of molecular structures otherwise unachievable.

\footnotetext{
a. Instituto de Ciencia Molecular (ICMol), Universidad de Valencia, c/Catedrático José Beltrán, 2, 46980 Paterna, Spain

b. Institut Lavoisier CNRS UMR 8180, Université de Versailles St Quentin-en-Yvelines, 45, Av. des Etats Unis, 78035 Versailles Cedex, France

c. Instituto de Tecnología Química (UPV-CSIC), Universidad Politécnica de ValenciaConsejo Superior de Investigaciones Científicas, Av. de los Naranjos s/n, 46022 Valencia, Spain

+ Footnotes relating to the title and/or authors should appear here.

Electronic Supplementary Information (ESI) available: additional figures and crystallographic data. See DOI: 10.1039/x0xx00000x
}

Very recently, we have exploited this concept of networked cages with the presence of latent porosity to develop unconventional crystalline solids capable of gas physisorption despite the absence of channels. ${ }^{12}$ The formation of compartmentalized coordination polymers (hereafter CCP) has resulted in high sorption selectivities towards different mixtures of gases. ${ }^{13}$ Furthermore, we have used as metallic centers $\mathrm{Fe}^{2+}$ ions able to undergo a thermal spin-crossover (SCO) transition. This feature has made these coordination polymers of interest in molecular magnetism since the confinement of the gas molecules in a restricted space has allowed switching the magnetic properties through gas sorption. These magnetic materials (CCP-1 and CCP-2) present infinite chains in which adjacent $\mathrm{Fe}^{\text {Il }}$ centers are linked by three organic ligand forming discrete voids of 135 $\AA^{3}$ due to the syn-conformation of the ligands. Up to one molecule of $\mathrm{CO}_{2}$ can be located in each void at 1 bar and 298 K. Thus, CCP-1 and CCP-2 are coordination polymers that present features typical of coordination cages.

In this work we have prepared isostructural analogues to these coordination polymers by extending the length of the flexible organic ligand with the purpose of augmenting the gas sorption capacity. Thus, we have synthesized two Fe" compartmentalized coordination polymers of formula $\left[\mathrm{Fe}(\mathrm{btzbp})_{3}\right]\left(\mathrm{ClO}_{4}\right)_{2} \quad(\mathrm{CCP}-3)$ and $\left[\mathrm{Fe}(\mathrm{btzbp}){ }_{(\mathrm{BF}}\right)_{2} \quad$ (CCP-4) $\left(\right.$ btzbp $=4,4^{\prime}$-bis $((1 \mathrm{H}$-tetrazol-1-yl)methyl)-1,1'-biphenyl) which present discrete voids of $257 \AA^{3}$. Gas sorption measurements show that two molecules of $\mathrm{CO}_{2}$ can be loaded in each void at 1 bar and $298 \mathrm{~K}$. In addition, both compounds present spin transition centred at 195 and $199 \mathrm{~K}$ respectively, similar to the observed in CCP-1 and CCP-2, although different magnetic behaviour has been observed depending on the number of $\mathrm{CO}_{2}$ molecules that are physisorbed.

\section{Experimental section}

\section{Materials}

All reagents and solvents were commercially available and used without further purification.

Synthesis of the ligand (btzbp). The ligand ligand 4,4'bis((1H-tetrazol-1-yl)methyl)-1,1'-biphenyl (btzbp) was synthesized following a three-step synthesis: 
a) 4,4'-bis(azidomethyl)-1,1'-biphenyl: A mixture of sodium azide $(1.0 \mathrm{~g}, 15 \mathrm{mmol})$ and 4,4'-bis(bromomethyl)1,1'-biphenyl ( $3.4 \mathrm{~g}, 10 \mathrm{mmol}$ ) was dissolved in $15 \mathrm{~mL}$ of dry DMF and stirred at $60{ }^{\circ} \mathrm{C}$ for 10 hours. Then, $100 \mathrm{~mL}$ of water was added and the product was extracted with ether $(3 \times 10$ $\mathrm{mL}$ ). The organic layer was washed three times with water (3 x $10 \mathrm{~mL}$ ). Yield $80 \%$. IR ( $\mathrm{KBr}$ disk) v $2109.5 \mathrm{~cm}^{-1}\left(\mathrm{~N}_{3}\right) ;{ }^{1} \mathrm{H}-\mathrm{NMR}$ (300 MHz, DMSO): $\delta 4.5$ (4 H, $\left.\mathrm{CH}_{2}-\mathrm{N}_{3}\right), 7.5(4 \mathrm{H}, \mathrm{CH}$ aromatic), 7.8 (4 H, CH aromatic).

b) [1,1'-biphenyl]-4,4'-diyldimethanamine: 4,4'bis(azidomethyl)-1,1'-biphenyl (1.5 g, $5.7 \mathrm{mmol})$ was dissolved in $25 \mathrm{~mL}$ of dry THF and added dropwise to a stirring solution of $\mathrm{LiAlH}_{4}(0.75 \mathrm{~g})$ in $25 \mathrm{~mL}$ of dry THF cooled to $0{ }^{\circ} \mathrm{C}$ under $\mathrm{Ar}$ atmosphere. When the addition was complete, the solution was heated to reflux with stirring for $4 \mathrm{~h}$. Then, the excess of $\mathrm{LiAlH}_{4}$ was quenched by slowly adding $0.75 \mathrm{~mL}$ of water, followed by $0.75 \mathrm{~mL}$ of a $15 \%$ aqueous $\mathrm{NaOH}$ solution and finally $2.25 \mathrm{~mL}$ of water with the suspension kept at $0{ }^{\circ} \mathrm{C}$ under constant stirring. The crude product was filtered and washed with ethyl acetate and dried under reduced pressure to yield the pure product. Yield $82 \%$. IR ( $\mathrm{KBr}$ disk) $\mathrm{N}_{3}$ band was not observed.

c) 4,4'-bis((1H-tetrazol-1-yl)methyl)-1,1'-biphenyl: A mixture of [1,1'-biphenyl]-4,4'-diyldimethanamine (1.5 g, 7.1 mmol), sodium azide $(0.92 \mathrm{~g}, 14.1 \mathrm{mmol})$ and triethyl orthoformate (12 mL, $69.3 \mathrm{mmol}$ ) was dissolved in $20 \mathrm{~mL}$ of acetic acid under Ar atmosphere and heated $90{ }^{\circ} \mathrm{C}$ for 4 days. After cooling to room temperature, the solvent was evaporated and the solid was washed with methanol to yield the pure product. Yield $74 \% .{ }^{1} \mathrm{H}-\mathrm{NMR}(300 \mathrm{MHz}, \mathrm{DMSO}): \delta$ $5.8\left(4 \mathrm{H}, \mathrm{CH}_{2}\right), 7.4(4 \mathrm{H}, \mathrm{CH}$ aromatic), $7.7(4 \mathrm{H}, \mathrm{CH}$ aromatic), $9.6(2 \mathrm{H}, \mathrm{CH}$ tetrazole).

Synthesis of $\left[\mathrm{Fe}(\mathrm{btzbp})_{3}\right]\left(\mathrm{ClO}_{4}\right)_{2} \quad$ (CCP-3). A solution of $\mathrm{Fe}\left(\mathrm{ClO}_{4}\right)_{2} \cdot \mathrm{xH}_{2} \mathrm{O}(28.0 \mathrm{mg})$ in $2 \mathrm{~mL}$ of $\mathrm{MeCN}$ was added into a solution of btzbp (63.6 mg, $0.2 \mathrm{mmol}$ ) in $24 \mathrm{~mL}$ of MeCN. A white crystalline precipitate appeared after a few days. The white powder was filtered and washed with MeCN. Phase purity was established by $\mathrm{X}$-ray powder diffraction (vide infra). Yield $61 \%$. Anal. calc. $\mathrm{C}_{48} \mathrm{H}_{42} \mathrm{FeN}_{24} \mathrm{Cl}_{2} \mathrm{O}_{8}$ (1209.75): C, 47.66; H, 3.50; N, 27.79 \%. Found: C, 47.50; H, 3.48; N, 26.94 $\%$.

Synthesis of $\left[\mathrm{Fe}(\mathrm{btzbp})_{3}\right]\left(\mathrm{BF}_{4}\right)_{2} \quad(\mathrm{CCP}-4)$. A solution of $\mathrm{Fe}\left(\mathrm{BF}_{4}\right)_{2} \cdot \mathrm{xH}_{2} \mathrm{O}(33.8 \mathrm{mg})$ in $2 \mathrm{~mL}$ of $\mathrm{MeCN}$ was added into a solution of btzbp (63.6 mg, $0.2 \mathrm{mmol})$ in $24 \mathrm{~mL}$ of MeCN. A white crystalline precipitate appeared after a few days. The white powder was filtered and washed with MeCN. Phase purity was established by $\mathrm{X}$-ray powder diffraction (vide infra). Yield $70 \%$. Anal. calc. $\mathrm{C}_{48} \mathrm{H}_{42} \mathrm{FeN}_{24} \mathrm{~B}_{2} \mathrm{~F}_{8}$ (1184.53): C, 48.67; H, 3.57; N, 28.38 \%. Found: C, 47.86; H, 3.54; N, 27.47 $\%$.

\section{Single crystal diffraction}

A single crystal of CCP-4 was mounted on a glass fibre using a viscous hydrocarbon oil to coat the crystal and then transferred directly to the cold nitrogen stream for data collection. X-ray data was collected on the same crystal at
$240 \mathrm{~K}$ (CCP-4-HS) and then at $120 \mathrm{~K}$ (CCP-4-LS) on a Supernova diffractometer equipped with a graphitemonochromated Enhance (Mo) X-ray Source $(\lambda=0.71073 \AA$ ). The program CrysAlisPro, Oxford Diffraction Ltd., was used for unit cell determinations and data reduction. Empirical absorption correction was performed using spherical harmonics, implemented in the SCALE3 ABSPACK scaling algorithm. Crystal structures were solved and refined against all $F^{2}$ values using the SHELXTL suite of programs. ${ }^{14}$ Due to the tiny size of the crystal (and thus weak diffraction data), only the Fe atoms were refined anisotropically. The disordered anions were modelled over two orientations with fixed thermal parameters, with occupancies 50:50. Hydrogen atoms were placed in calculated positions that were refined using idealized geometries (riding model) and assigned fixed isotropic displacement parameters. CCDC-1440481 (CCP-4HS) and -1440482 (CCP-4-LS) contain the supplementary crystallographic data for this paper. A summary of selected bond distances and angles is provided in Table S1, and a summary of the data collection and structure refinements is provided in Table S3.

\section{X-ray powder diffraction}

Polycrystalline samples of CCP-3 and CCP-4 were lightly ground in an agate mortar and pestle and filled into $0.5 \mathrm{~mm}$ borosilicate capillaries prior to being mounted and aligned on a Empyrean PANalytical powder diffractometer, using $\mathrm{Cu}$ K $\alpha$ radiation $(\lambda=1.54056 \AA)$. For each sample, two repeated measurements were collected at room temperature $(2 \theta=2$ $60^{\circ}$ ) and merged in a single diffractogram. Pawley refinements ${ }^{15}$ were performed using the TOPAS computer program $^{16}$ and revealed an excellent fit to a one-phase model for compounds CCP-3 $\left(R_{w p}=0.0218\right.$; GOF $\left.=1.502\right)$ and CCP-4 ( $R_{w p}=0.0239 ;$ GOF = 1.225), indicating the absence of any other detectable crystalline phases.

\section{Magnetic measurements}

Magnetic susceptibility measurements were carried out on single-phased polycrystalline samples with a Quantum Design MPMS-XL-5 SQUID susceptometer. The susceptibility data were all collected at $1 \mathrm{~K} \cdot \mathrm{min}^{-1}$, with an applied field of $0.1 \mathrm{~T}$. Magnetic susceptibility measurements of the gas loaded systems were performed by sealing a glass tube with $10 \mathrm{mg}$ of CCP-4 and a known amount of $\mathrm{CO}_{2}$, or after heating a loaded sample to remove the $\mathrm{CO}_{2}$ molecules. Prior to the gas loading, the samples were activated by heating in situ at $150^{\circ} \mathrm{C}$ for $3 \mathrm{~h}$ under vacuum. In order to discard leak effects, the experiment was repeated twice obtaining the same results.

\section{Gas sorption isotherms}

High-resolution isotherms were measured at a series of temperatures in a Micromeritics ASAP 2010 volumetric instrument using approximately $150 \mathrm{mg}$ of CCP-4 as a powder. The sample was placed in a sample holder that was immersed into a liquid recirculating thermostatic bath (Julabo FP40-HL) able to control the adsorption temperature with accuracy better than $0.05 \mathrm{~K}$. Prior to adsorption 
experiments, the sample was outgassed at $423 \mathrm{~K}$ for 6 hours under turbomolecular high vacuum. To avoid He adsorption after free space volume determination the sample was outgassed for 1 hour at $423 \mathrm{~K}$ prior to gas adsorption. We have applied the Virial isotherm for fitting experimental data points and it was found that a fourth grade polynomial is able to properly describe the $\mathrm{CO}_{2}$ isotherms. The heat of adsorption was calculated accordingly to the ClausiusClapeyron equation from the isotherms taken at different temperatures.

\section{Surface deposition studies}

Two different substrates were studied for this purpose: silicon and quartz substrates. The same procedure was carried out in the two cases. The substrates were washed by immersion in an acid piranha solution for three minutes and subsequent washing with water. Then, the clean substrates were introduced in a fresh solution containing both the ligand and the metal. After few days the substrate was removed from the solution, washed with acetonitrile and dried over nitrogen stream.

SEM studies were performed on a Hitachi S-4800 microscope operating at an accelerating voltage of $10 \mathrm{kV}$ after metallization of the samples (Au-Pd).

\section{Results and discussion}

\section{Structural characterization}

We have previously reported ${ }^{12,13}$ the formation of two compartmentalized coordination polymers (CCP) which present thermal spin transition with the use of 1,4bis(tetrazol-1-ylmethyl)benzene (hereafter btzx) as ligand. With the aim of increasing the $\mathrm{CO}_{2}$ sorption capacity of in these CCPs, we have designed an organic ligand that extends the intermetallic distance by adding an extra benzene ring in its skeleton. Thus, the ligand 4,4'-bis( $(1 \mathrm{H}$-tetrazol-1yl)methyl)-1,1'-biphenyl (hereafter btzbp, see Scheme 1) is $4.5 \AA$ A larger than the previously used btzx.

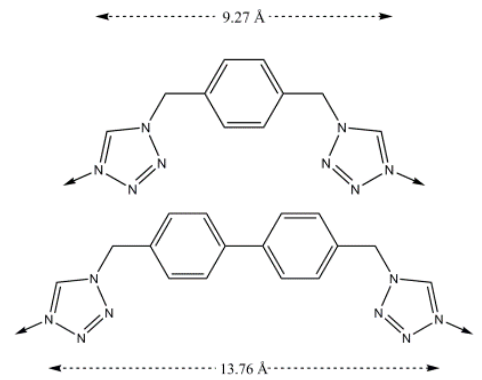

Scheme 1. Chemical structures of the ligands btzx (top) and btzbp (bottom). The dashed arrows represent the distances between the two coordinating nitrogens.

The reaction of btzbp with two differents $\mathrm{Fe}^{\text {Il }}$ salts in refluxing $\mathrm{MeCN}$ during $4 \mathrm{~h}$ affords two coordination polymers, CCP-3 and CCP-4, with general formula $\left[\mathrm{Fe}(\mathrm{btzbp})_{3}\right](\mathrm{X})_{2}\left(\mathrm{X}=\mathrm{ClO}_{4}\right.$ or $\left.\mathrm{BF}_{4}\right)$. By the use of microwave irradiation ${ }^{17}\left(82^{\circ} \mathrm{C}\right)$, the synthesis time could be significantly reduced to $5 \mathrm{~min}$. Nevertheless, a higher quality material was obtained from the reflux reaction. Structural analysis of CCP4 in the high-spin (HS) and the low-spin (LS) states were determined using the same single crystal at 240 and $120 \mathrm{~K}$, respectively. In both states, CCP-4 crystallizes in the space group $P 6_{3}$, and is composed of $\left[\mathrm{Fe}(\mathrm{btzbp})_{3}\right]^{2+}$ units that form one-dimensional chains that run parallel to crystallographic c-axis with Fe $\cdots$ Fe distances of $16.1445 \AA$ at $240 \mathrm{~K}$ (Figure 1). The $\mathrm{Fe}^{\text {Il }}$ centers are triply bridged by the btzbp organic ligands, in which the two phenyl rings are not coplanar, causing a reduction in symmetry from $P G_{3} / m$ to $P 6_{3}$ when comparing with CCP-1 and CCP-2 that contains the shorter btzx ligand. The $\mathrm{BF}_{4}{ }^{-}$counterions arrange coplanar to the $\mathrm{Fe}^{\mathrm{II}}$ atoms and separate the different chains, which are closely packed affording a framework with no permanent channels but with internal discrete voids of $257 \AA^{3}$ at $240 \mathrm{~K}\left(235 \AA^{3}\right.$ at $120 \mathrm{~K})$, which are practically double in size to those found in CCP-1 and CCP-2 $\left(135 \AA^{3}\right)$. Each Fe"l center, which lies on the 3-fold axis, is octahedrally coordinated by six tetrazoles nitrogen atoms from btzbp ligands. The $\mathrm{Fe}-\mathrm{N}$ distances are 2.16(3) $\AA$ at $240 \mathrm{~K}$ while these are 2.01(3) $\AA$ at $120 \mathrm{~K}$. The distances are in good agreement with $\mathrm{Fe}-\mathrm{N}$ distances at $\mathrm{HS}$ and LS respectively in Fe-tetrazolate systems. ${ }^{18}$ The change in the length corresponds to the change in the spin of the Fe (lower Fe-N distances at LS), which is also accompanied by a colour change of the crystal from colorless (240 K) to pink (120 K).

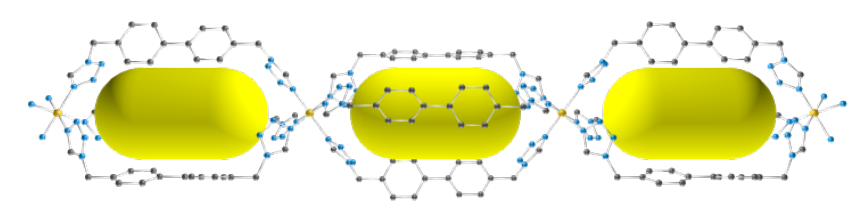

Figure 1. Crystal structure of CCP-4 viewed along the $b$-axis. The $\mathrm{BF}_{4}^{-}$anions and hydrogen atoms have been removed for clarity. Key: Fe, orange; C, gray; N, blue. The yellow ellipsoids are placed in the structure to represent the empty space of the internal voids.

\section{Sorption properties}

Although the crystal structures of CCP-3 and CCP-4 present no permanent channels, we have recently reported ${ }^{13}$ the mechanism by which the analogues with shorter ligands, CCP-1 and CCP-2, can load different gases in their internal voids. Due to the structural similarity, we have explored the sorption capabilities of CCP-4.
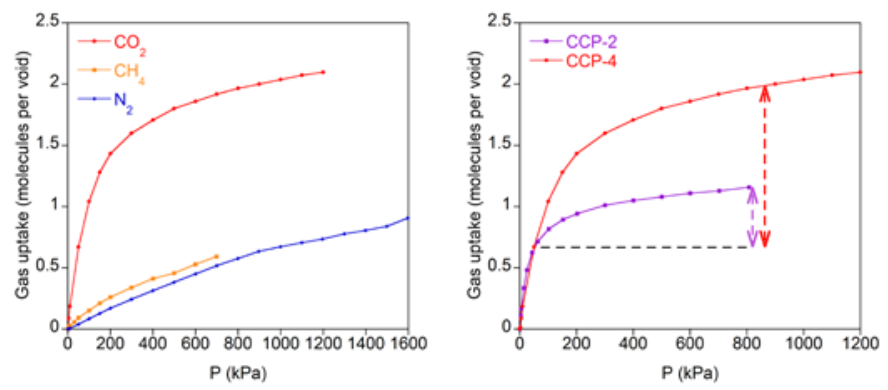
a)

b)

Figure 2. a) Gas sorption isotherms at $298 \mathrm{~K}$ for $\mathrm{CO}_{2}, \mathrm{CH}_{4}$ and $\mathrm{N}_{2}$ for CCP-4 b) comparison between the capability of CCP-2 and $\mathrm{CCP}-4$ in $\mathrm{CO}_{2}$ uptake. The double arrowed lines indicate the working capacities of each system.

Single-components isotherms have been applied to determine the loading capacities towards $\mathrm{CO}_{2}, \mathrm{~N}_{2}$ and $\mathrm{CH}_{4}$ at $298 \mathrm{~K}$. The isosteric heats of adsorption on CCP-2 and CCP-4 for $\mathrm{CO}_{2}$, shown in Figure $\mathrm{S} 4$, were calculated from the Clausius-Clapeyron equation using adsorption data collected at $283,298,313$ and $333 \mathrm{~K}$ to quantify the adsorption affinity for $\mathrm{CO}_{2}$. The isosteric heat of adsorption for $\mathrm{CO}_{2}$ at zero coverage on CCP-4 was estimated to be $16.3 \mathrm{~kJ} \cdot \mathrm{mol}^{-1}$, which is slightly lower than that found in CCP-2 $\left(22.0 \mathrm{~kJ} \cdot \mathrm{mol}^{-1}\right)$.

As shown in Figure 2, CCP-4 is able to adsorb two molecules of $\mathrm{CO}_{2}$ in each discrete compartment, at atmospheric conditions (at 12 bar and $298 \mathrm{~K}$ ), whereas a much lower content of $\mathrm{N}_{2}$ and $\mathrm{CH}_{4}$ is observed. By contrast, CCP-1 and CCP-2 have a $\mathrm{CO}_{2}$ maximum capacity of one molecule per void. Therefore, we have successfully increased the content in each void by chemical design. This has a direct impact on the working capacity, ${ }^{19}$ here defined as the difference of the uptake at $800 \mathrm{kPa}$ minus the uptake $50 \mathrm{kPa}$. As shown in Figure $2 \mathrm{~b}$, the $\mathrm{CO}_{2}$ working capacity of $\mathrm{CCP}-4$ is more than twice than that of CCP-2, due to the larger adsorption capacity as well as the lower isosteric heat of adsorption of CCP-4 compared to CCP-2. In addition, the use of a larger ligand does not penalize the high volumetric sorption observed in CCP-1 and CCP-2, as the dense nature of the material is maintained. At 1 bar and $298 \mathrm{~K}$ the system is able to storage $29 \mathrm{~mL}$ of $\mathrm{CO}_{2}$ per $\mathrm{mL}$ of material, which is similar in value to the storage capacity of MIL-100 and greater than that presented by MOF-5, although lower than the storagerecord of MOF-74-Mg (see Table 1). This is a consequence of the high density of compartmentalized coordination polymers, which contrasts with the situation encountered in the vast majority of MOFs, which have a low density value due to the presence of large channels in the structure. ${ }^{20}$

Table 1. Volumetric $\mathrm{CO}_{2}$ capacity for different systems

\begin{tabular}{ccc}
\hline Compound & $\begin{array}{c}\mathrm{CO}_{2} \text { sorption } \\
\left(\mathrm{mL} \mathrm{CO}_{2} \cdot \mathrm{mL}^{-1} \mathrm{MOF}\right)\end{array}$ & Reference \\
\hline CCP-4 & 29 & this work \\
CCP-1 & 29 & 13 \\
CCP-2 & 27 & 13 \\
MIL-100 & 28 & 21 \\
MOF-5 & 11 & 22 \\
MOF-74-Mg & 128 & 23 \\
\hline
\end{tabular}

\section{Magnetic properties}

Magnetic susceptibility measurements performed on polycrystalline samples of CCP-3 and CCP-4 show that both compounds present a spin transition centred at 195 and 199 $\mathrm{K}$ respectively, similar to that observed in CCP-1 and CCP-2 (200 K), albeit no hysteresis is found. This behaviour can be ascribed to some loss in cooperativity due to the longer distance between each iron centre in the crystal. However, it is surprisingly to observe that the abruptness in the transition is maintained with metal centres separated $16 \AA$. This is probably related to the polymeric structure of the materials, with low dimensionality, and the existence of a triple bridge between the iron centres. As can be observed in Figure 3 , the use of the larger ligand also causes an increase in the residual high-spin fraction, which is ca. $20 \%$.
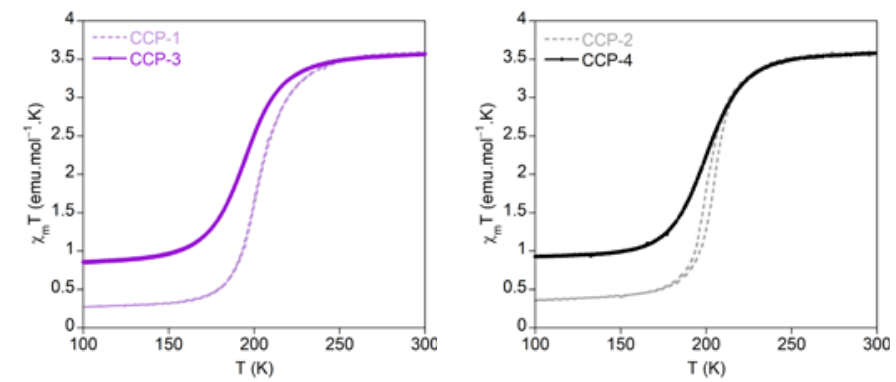

Figure 3. Temperature dependence of the product of the magnetic susceptibility times the temperature $\left(\chi_{\mathrm{m}} \mathrm{T}\right)$ for CCP3 (purple) and CCP-4 (black), compared with those of CCP-1 and CCP-2 (dashed lines).

We have previously studied two different approaches of modulating the spin transition of CCP-1 with chemical stimuli: i) the inclusion of $\mathrm{CO}_{2}$ molecules shifts the transition temperature to higher temperature; ${ }^{12}$ ii) the use of ligand mixtures subtly modifies the transition temperature and also new transitions at lower temperatures are generated. ${ }^{24}$

In the present case the perturbation exerted by the physisorption of $\mathrm{CO}_{2}$ has been studied for CCP-4. Upon inclusion of one molecule of $\mathrm{CO}_{2}$ in each internal void, a shift of $\mathrm{T}_{1 / 2}$ from $199 \mathrm{~K}$ to $206 \mathrm{~K}$ has been observed (Figure 4). Thus, physisorption of $\mathrm{CO}_{2}$ stabilizes the LS state, as was also observed in CCP-1 and CCP-2, due to the interaction between $\mathrm{CO}_{2}$ and the cationic framework. Interestingly, upon additional $\mathrm{CO}_{2}$ loading (two molecules in each void), no further increase of $T_{1 / 2}$ is observed. On the contrary, a reduction of $\mathrm{T}_{1 / 2}$ occurs, reaching $202 \mathrm{~K}$. This behaviour could be caused by gas-gas interaction inside the void of the compartmentalized coordination polymer. The two $\mathrm{CO}_{2}$ molecules interact with each other, thus decreasing the interaction between the gas and the framework, and therefore reducing the effects in $T_{1 / 2}$.
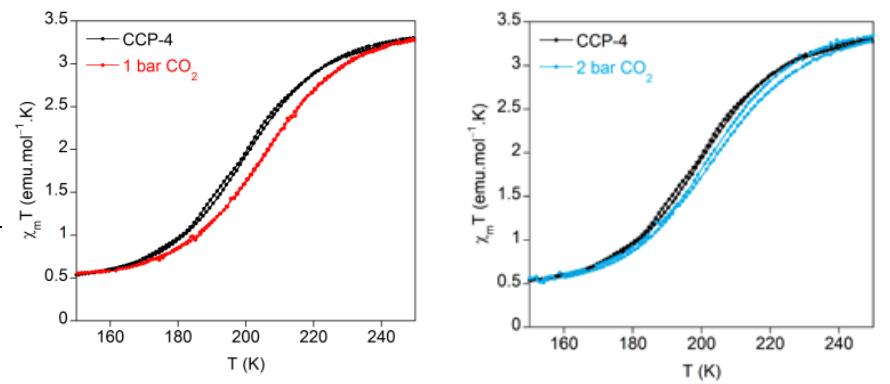
Figure 4. Temperature dependence product of the magnetic susceptibility times the temperature $\left(\chi_{m} T\right)$ for CCP-4 loaded with one (red line) and two (blue line) molecules of $\mathrm{CO}_{2}$ in each void.

\section{Surface deposition}

Surface deposition studies have been carried out as preliminary tests in order to examine the possible preparation of membranes for $\mathrm{CO}_{2}$ capture or selective separation of gases. ${ }^{25}$ For this purpose, we have analysed the deposition of CCP-4 on two different substrates, quartz and silicon by drop casting and we concluded than the choice of substrate plays an important role in the result. The deposition is not complete on quartz substrate, with only some areas covered. However, on these regions we were able to find some hexagonal micro crystals of the system (Figure 5a). On the other hand, when using a silicon substrate, a complete coverage is achieved, with each silicon substrate covered by a layer of $400 \mu \mathrm{m}$ of micrometric particles with irregular shapes (Figure 5b). Confirmation of the formation of CCP-4 on top of the substrate was achieved with grazing incidence X-ray diffraction (GIXRD). As Figure 6 shows, the same diffractogram is obtained for the film and the polycrystalline powder, thus demonstrating the formation deposition of the compartmentalized coordination polymer. Additionally, the magnetic behaviour of the deposited material shows that the spin transition is maintained (Figure S8).

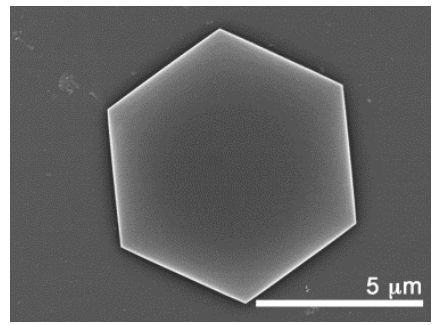

a)

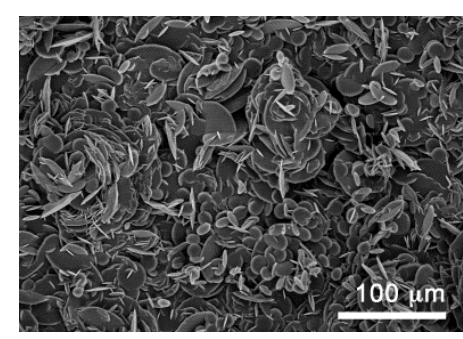

b)
Figure 5. a) Hexagonal microcrystal of CCP-4 on a quartz substrate; b) irregular micrometric particles of CCP-4 covering all the silicon substrate.

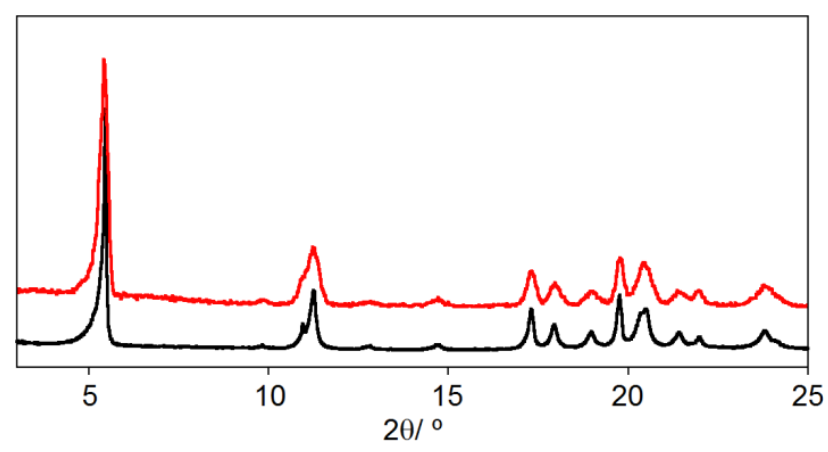

Figure 6. XRPD of polycrystalline powder of CCP-4 (black line) and GIXRD of the film for the same material (red line).

\section{Conclusions}

In this work we have prepared two compartmentalized coordination polymers with general formula $\left[\mathrm{Fe}(\mathrm{btzbp})_{3}\right](\mathrm{X})_{2}$ $\left(\mathrm{X}=\mathrm{ClO}_{4}\right.$ or $\left.\mathrm{BF}_{4}\right)$, which can be of interest in two different areas: MOFs and molecular magnetism. In the former area we have shown that these crystalline solids possess internal cavities that have been proved to be suited for accommodating up to two molecules of $\mathrm{CO}_{2}$ each. In molecular magnetism we have shown that these polymers are examples of multifunctional magnetic materials responding to a chemical stimulus. In fact, they present spincrossover phenomena which can be tuned by sorption of $\mathrm{CO}_{2}$. Thus, an increase in the transition temperature at low loadings from $T_{1 / 2}=199 \mathrm{~K}$ to $T_{1 / 2}=206 \mathrm{~K}$ has been observed as a result of the gas-framework interaction. Upon further loading, the presence gas-gas interactions reduce the effects on the framework causing a reduction in the shift of the transition temperature, being $\mathrm{T}_{1 / 2}=202 \mathrm{~K}$. Finally, the formation of thin films opens the possibility to easily process these materials in the form of membranes to be used in gas separation processes.

\section{Acknowledgements}

Financial support from the Spanish MINECO (CTQ2014-59209P, MAT2014-56143-R and MAT2012-38567-C02-01), the Generalitat Valenciana (Prometeo and ISIC-Nano programs) and the VLC/Campus Program is gratefully acknowledged. We thank the Spanish government for the provision of a Severo Ochoa project (SEV-2012-0267) and a María de Maeztu project (MDM-2015-0538). M.G.-M. thanks MICINN for a predoctoral FPU grant and the EU for a Marie Sklodowska-Curie postdoctoral fellowship (H2020-MSCA-IF-EF-658224). N.C.G. thanks the Generalitat Valenciana for a $\mathrm{Val}-\mathrm{i}+\mathrm{d}$ predoctoral fellowship. J. M. Martínez-Agudo and G. Agustí from the University of Valencia are gratefully acknowledged for magnetic measurements.

\section{Notes and references}

\# Crystal data for CCP-4. [ $\left.\mathrm{Fe}\left(\mathrm{C}_{16} \mathrm{H}_{14} \mathrm{~N}_{8}\right)_{3}\right]\left(\mathrm{BF}_{4}\right)_{2}$. Space group $P 6_{3}$, $\mathrm{Z}=2, M=1184.53$. HS: $\mathrm{T}=240(2) \mathrm{K}, a=b=10.3650$ (10) $\AA$, $c=$ $32.289(5) \AA, V=3004.2(6) \AA^{3} . R 1=0.2271$ and $w R 2=0.5406$. LS: $\mathrm{T}=120(2) \mathrm{K}, a=b=10.2235(14) \AA, c=31.903(8) \AA, \mathrm{V}=$ $2887.8(11) \AA^{3} . R 1=0.2157$ and $w R 2=0.5271$.

1 (a) B. F. Hoskins and R. J. Robson, J. Am. Chem. Soc., 1989, 111, 5962; (b) B. F. Hoskins and R. J. Robson, J. Am. Chem. Soc., 1990, 112, 1546.

2 E. Coronado, M. Giménez-Marqués, G. Mínguez Espallargas and L. Brammer, Nat. Commun., 2012, 3, 828 .

3 See special issues on MOFs: Chem. Rev., 2012, 112, 673; Chem. Soc. Rev., 2014, 43, 5415.

4 (a) H. Furukawa, K. E. Cordova, M. O'Keeffe and O. M. Yaghi, Science, 2013, 341, 6149; (b) A. G. Slater and A. I. Cooper, Science, 2015, 348, 6238.

5 Z. Hu, B. J. Deibert and J. Li, Chem. Soc. Rev., 2014, 43, 5815.

6 E. Coronado and G. Mínguez Espallargas, Chem. Soc. Rev., 2013, 42, 1525

7 J.-R. Li, R. J. Kuppler and H.-C. Zhou, Chem. Soc. Rev., 2009, 38, 1477. 
8 L. J. Murray, M. Dincă and J. R. Long, Chem. Soc. Rev., 2009, 38, 1294.

9 M. Giménez-Marqués, T. Hidalgo, C. Serre and P. Horcajada, Coord. Chem. Rev., 2015, In press

10 (a) M. J. Smulders, I. A. Riddell, C. Browne and J. R. Nitschke, Chem. Soc. Rev., 2013, 42, 1728; (b) T. R. Cook and P. J. Stang, Chem. Rev., 2015, 115, 7001.

11 (a) Y. Inokuma, S. Yoshioka, J. Ariyoshi, T. Arai, Y. Hitora, K. Takada, S. Matsunaga, K. Rissanen and M. Fujita, Nature, 2013, 495, 461; (b) S. Yoshioka, Y. Inokuma, M. Hoshino, T. Sato and M. Fujita, Chem. Sci., 2015, 6, 3765.

12 E. Coronado, M. Giménez-Marqués, G. Mínguez Espallargas, F. Rey and I. J. Vitórica-Yrezábal, J. Am. Chem. Soc., 2013, 135, 15986.

13 M. Giménez-Marqués, N. Calvo Galve, M. Palomino, S Valencia, F. Rey, G. Sastre, I. J. Vitórica-Yrezábal, M. JiménezRuiz, J. A. Rodríguez-Velamazán, M. A. González, E. Coronado and G. Mínguez Espallargas, submitted

14 G. M. Sheldrick, Acta Crystallogr., Sect. A: Found. Crystallogr., 2008, 64, 112.

15 G. S. Pawley, J. Appl. Crystallogr., 1981, 14, 357.

16 A. A. Coelho, TOPAS-Academic, Version 4.1, 2007, see http://www.topas-academic.net.

17 J. Klinowski, F. A. Almeida Paz, P. Silva and J. Rocha, Dalton Trans., 2011, 40, 321.

18 G. Aromí, L. A. Barrios, O. Roubeau and P. Gamez, Coord. Chem. Rev., 2011, 255, 485.

19 M. Palomino, A. Corma, F. Rey, S. Valencia, Langmuir 2010, 26, 1910.

20 R. E. Morris and P. S. Wheatley, Angew. Chem. Int. Ed., 2008, 47, 4966.

21 J. A. Mason, T. M. McDonald, T. Bae, J. E. Bachman, K. Sumida, J. J. Dutton, S. S. Kaye and J. R. Long, J. Am. Chem. Soc., 2015, 137, 4787

22 A. Ö. Yazaydin, R. Q. Snurr, T.-H. Park, K. Koh, J. Liu, M. D. LeVan, A. I. Benin, P. Jakubczak, M. Lanuza, D. B. Galloway, J. L. Low and R. R. Willis, J. Am. Chem. Soc., 2009, 131, 18198.

23 S. R. Caskey, A. G. Wong-Foy and A. J. Matzger, J. Am. Chem. Soc., 2008, 130, 10870.

24 N. Calvo Galve, E. Coronado, M. Giménez-Marqués and G. Mínguez Espallargas, Inorg. Chem., 2014, 53, 4482.

25 Z. Xiang and D. Cao, J. Mater. Chem. A, 2013, 1, 2691. 